\title{
Performance Evaluation of $1 / 2$ Rate Convolution Coding with Different Modulation Techniques for DS-CDMA System over Rician Channel
}

\author{
Dilip Mandloi \\ PG Scholar Department of ECE, \\ IES, IPS Academy, Indore [India]
}

\author{
Prateek Paliwal \\ PG Scholar Department of \\ ECE, SIMS, Indore [India]
}

\author{
Deepali Yadav \\ PG Scholar Department of \\ ECE SIMS, Indore [India]
}

\begin{abstract}
In this paper we evaluate the performance of $1 / 2$ rate convolution coding with different modulation techniques such as Binary phase shift keying (BPSK), Quadrature phase shift keying (QPSK) and Quadrature amplitude modulation (QAM16) for direct sequence code division multiple access(DSCDMA) system using maximal ratio combining (MRC) and equal gain combining (EGC) diversity techniques over Rician fading channel. The performance of $1 / 2$ rate convolution coding with different modulation techniques are analyzed in terms of Bit error rate (BER) and Signal to noise ratio (SNR). Based on simulation results we have concluded that we obtain better gain in SNR performance when $1 / 2$ rate convolution coding is used with different modulation techniques.
\end{abstract}

\section{General Terms}

Modulation techniques, fading channel, Bit error rate (BER), Signal to noise ratio (SNR)

\section{Keywords}

Convolution coding, BPSK, QPSK, QAM-16, DS-CDMA, maximal ratio combining (MRC), equal gain combining (EGC), Rician channel

\section{INTRODUCTION}

Performance of DS-CDMA system suffers from multipath effects such as reflection, diffraction and scattering which results in signal fading which degrades the performance of DS-CDMA system [1]. Diversity techniques together with channel coding, is a popular technique to mitigate the effects of multipath fading in wireless communication [2]. When a direct line of sight path is available between the transmitter and receiver channel can be modelled as Rician channel for which the fading amplitude obey a Rician distribution. To reduce Rician fading, convolutional codes with Viterbi decoding and interleaving could be used [3]. The most important combining methods used to combine the signal which is received at the receiver from multiple paths in order to increase the overall received SNR are Selection combining (SC), Maximal ratio combining (MRC) and Equal gain combining (EGC) [4]. Among all these diversity combining techniques, MRC is the optimal technique at the cost of increased receiver complexity. EGC is an alternative technique that is used in practice because EGC is easier to implement compared to MRC and performance of EGC is comparable to MRC but better then SC [5].

In this paper we study the BER performance of $1 / 2$ rate convolutional coding for a DS-CDMA system with MRC and EGC diversity reception over Rician fading channel. Different modulation schemes namely BPSK, QPSK and QAM-16 are considered for modulation.

The paper is organized as follows: In Section 2, the model of the transmission system used is given. Simulation parameter and simulation results are discussed In Section 3. A brief conclusion is given in Section 4.

\section{SYSTEM MODEL}

\subsection{DS-CDMA System}

We consider a binary DS-CDMA system. The information rate is $R$ bits/sec and the bit interval is $T_{b}=1 / R \mathrm{sec}$. The available channel bandwidth is $B_{c} \mathrm{~Hz}$, where $B_{c} \gg R$.

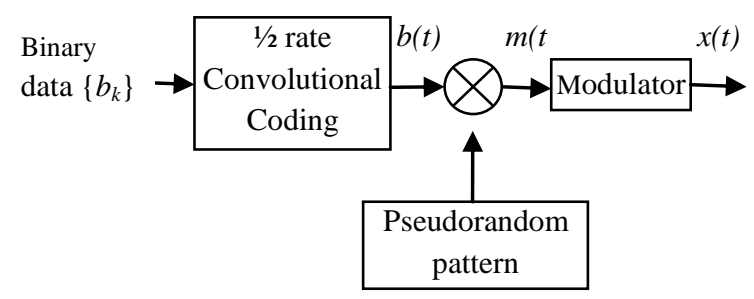

Fig 1: Transmitter structure

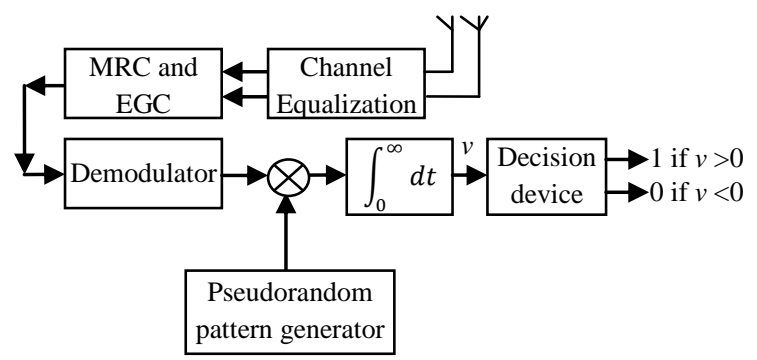

Fig 2: Receiver structure

At the modulator, the bandwidth of the information signal is expanded to $W=B_{c} H z$ by shifting the phase of the carrier pseudorandomly at a rate of $W$ times/sec according to the pattern of the PN generator. The information-bearing baseband signal is denoted as $v(t)$ and is expressed as

$$
v(t)=\sum_{n=-\infty}^{\infty} a_{n} g_{T}\left(t-n T_{b}\right)
$$

Where $\{a= \pm 1,-\infty<n>\infty\}$ and $g_{T}(t)$ is a rectangular pulse of duration $T_{b}$. This is multiplied by the signal is multiplied by the signal from the PN sequence generator, which expressed as

$$
c(t)=\sum_{n=-\infty}^{\infty} c_{n} p\left(t-n T_{c}\right)
$$

where $c_{n}$ represents the binary PN code sequence of \pm 1 'S and $p(t)$ is a rectangular pulse of duration $T_{c}$. This multiplication operation serves to spread the bandwidth of the information bearing into the wider bandwidth occupied by PN generator 
signal $c(t)$. The resulting transmitted signal $u(t)$ is thus given by

$$
u(t)=A_{c} v(t) c(t) \cos 2 \pi f_{c} t
$$

\subsection{Convolution coding}

Convolutional coding is used reduce the bit errors introduced by transmission of a modulated signal through a wireless channel. A convolutional code generates coded symbols by passing the information bits through a linear finite-state shift register. The shift register consists of $\mathrm{K}$ stages with $\mathrm{k}$ bits per stage. There are $\mathrm{n}$ binary addition operators with inputs taken from all $\mathrm{K}$ stages these operators produce a codeword of length $\mathrm{n}$ for each $\mathrm{k}$-bit input sequence. Specifically, the binary input data is shifted into each stage of the shift register $\mathrm{k}$ bits at a time, and each of these shifts produces a coded sequence of length $n$ [6]. The rate of the code is.

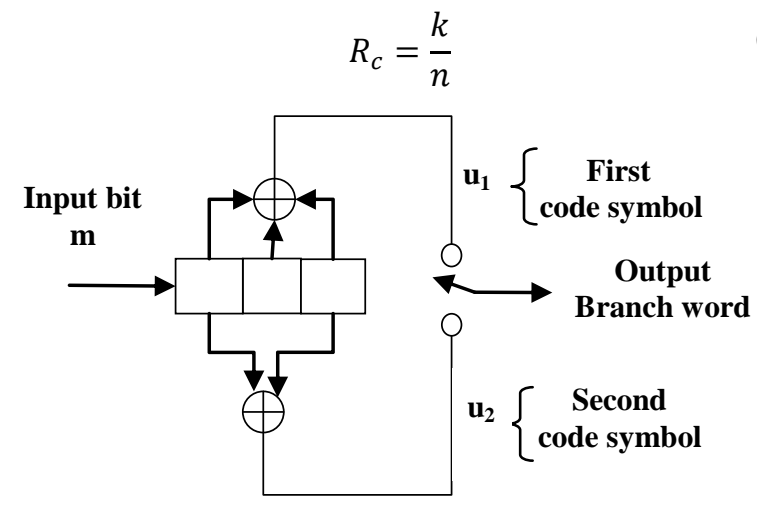

Fig 3: 1/2 rate convolutional encoder

Fig 3 shows a $1 / 2$ rate convolutional encoder. There are a number of techniques for decoding convolutional codes. The most important of these methods is the Viterbi algorithm which performs maximum likelihood decoding of convolutional codes.

\subsection{Digital modulation techniques}

Digital modulation is the process by which binary bits are transformed into waveforms that are compatible with the characteristics of the channel. In this paper we consider fallowing modulation techniques:

\subsubsection{Binary phase shift keying (BPSK)}

In BPSK modulator the carrier assumes one of two phases. Logic 1 produces no phase change and logic 0 produces an $180^{\circ}$ phase change [7]. The output waveform for BPSK modulator is shown in Fig.
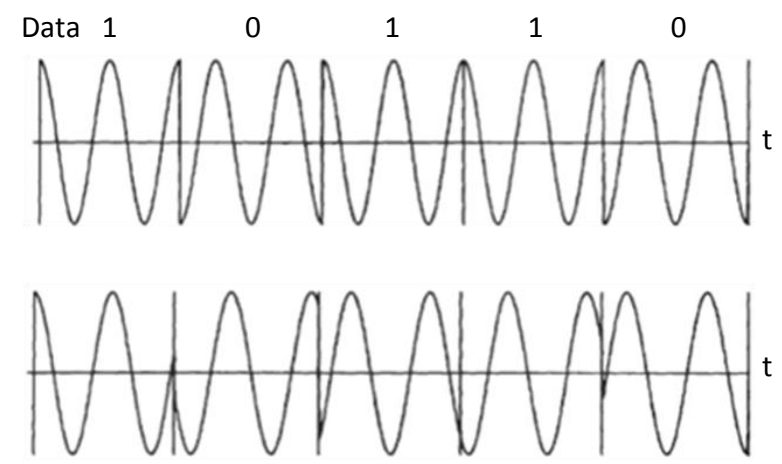

Fig 4: Binary phase shift keying

\subsubsection{Quadrature phase shift keying (QPSK)}

In QPSK modulator 2 bits are processed to produce a single phase change. In QPSK modulation each symbol consists of 2 bits, which are referred to as a dibit. Bandwidth efficiency of QPSK is twice as compared to the BPSK because two bits are transmitted in a single modulation symbol [7].

$$
\begin{array}{cc}
s_{i}(t)=\sqrt{\frac{2 E}{T}} \cos \left(2 \pi f_{c} t+\theta_{i}\right), & 0 \leq t \geq T_{b} \\
\theta_{i}=\frac{(2 i-1) \pi}{4} & i=1,2,3,4
\end{array}
$$

The initial phases produced by QPSK modulator are $\pi / 4$, $3 \pi / 4,5 \pi / 4$ and $7 \pi / 4$.

\subsubsection{Quadrature amplitude modulation(QAM)}

In M-ary PSK modulation, the amplitude of the transmitted signal was constrained to remain constant, thereby yielding a circular constellation. By allowing the amplitude to also vary with the phase, a new modulation scheme called quadrature amplitude modulation (QAM) is obtained [7]. Constellation diagram of 16- QAM is shown in Fig 5.

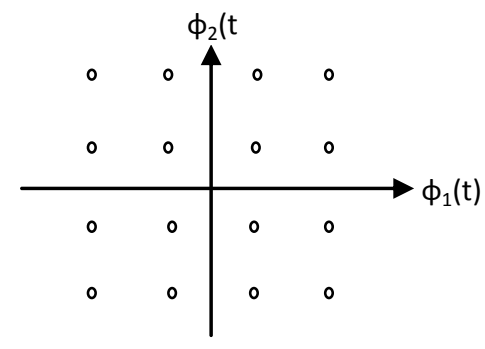

Fig 5: Constellation diagram of QAM-16

\subsection{Rician fading}

The Rician distribution is observed when a direct path is exist between the transmitter and the receiver in addition to the multipath components. Due to presence of direct LOS component amount of fading is reduced as compared to the Rayleigh fading [8].PDF of the Rician distribution is given by

$$
f(r)=\frac{r}{\sigma^{2}} \exp \left(-\frac{r^{2}+k_{d}^{2}}{2 \sigma^{2}}\right) I_{0}\left(\frac{r k_{d}}{\sigma^{2}}\right) \quad r \geq 0
$$

Rician distribution is described in terms of Rician factor $\mathrm{K}_{0}$, defined as

$$
K_{0(d b)}=10 \log _{10}\left(\frac{a_{0}^{2}}{2 \sigma^{2}}\right)
$$

The quantity $\mathrm{K}_{0}$ is a measure of the strength of the LOS component, and when $\mathrm{K}_{0} \rightarrow 0$, we have Rayleigh fading. As $\mathrm{K}_{0}$ increases, the fading in the channel declines.

\subsection{Diversity combining techniques}

Diversity techniques are used to reduce the effect of multipath signal fading which is generally occur due do multipath effect such as reflection, diffraction and scattering. Diversity combining techniques combine the multiple replicas of the same information-bearing signal which are received from different path at the receiver to increase the overall received SNR. In this paper we consider fallowing two type of diversity combining techniques: 


\subsubsection{Maximal Ratio Combining (MRC)}

In MRC the signals received from multiple path weighted according to their individual signal voltage to noise power ratios and then summed. Here, the individual signals must be co-phased before being summed. Maximal ratio combining produces an output SNR equal to the sum of the individual SNRs. Thus, it has the advantage of producing an output with an acceptable SNR even when none of the individual signals are themselves acceptable [7].

\subsubsection{Equal Gain Combining (EGC)}

The EGC receiver processes all the received replicas, weights them equally and then sums them to produce the decision statistic. In EGC receiver estimation of the channel carrier phase is required but the weights applied to each branch in the combiner are complex quantities whose amplitudes are all set to 1 . EGC provide comparable performance to the MRC with less receiver complexity [9].

\section{SIMULATION RESULTS}

In this section, we present and discuss the simulation results of the BER Vs SNR performance of $1 / 2$ rate convolutional coding with different modulation schemes (BPSK,QPSK and QAM-16) for a DS-CDMA system using MRC and EGC diversity reception over multipath Rician fading channel with Rician parameter $\mathrm{K}=1$ and $\mathrm{K}=5 \mathrm{db}$ through MATLAB. Simulation parameter is given in table 1.

\subsection{Bit Error Rate (BER)}

BER is defined as the rate at which errors occur in a transmission system due to noise, interference etc. To evaluate BER mathematically we take the ratio of number of errors to total number of bits transmitted

$$
B E R=\frac{\text { Number of Errors }}{\text { Total no of Bits Transmitted }}
$$

\subsection{Signal to Noise Ratio (SNR)}

The signal to noise ratio is a measure of the sensitivity performance of a receiver. SNR is defined as the ratio of signal power to noise power it is usually measured in decibel. The SNR mathematically can be expressed as follows:

$$
S N R_{d b}=10 \log _{10}\left(\frac{P_{\text {signal }}}{P_{\text {noise }}}\right)
$$

Table 1: Simulation Parameters

\begin{tabular}{|l|l|}
\hline Parameters & value \\
\hline Number of input data bits & $\mathbf{9 0 0}$ \\
\hline Chip rate & $\mathbf{8}$ Mbps \\
\hline Number of users & $\mathbf{1}$ \\
\hline Channel coding & Convolutional coding \\
\hline Code rate & $\mathbf{1 / 2}$ \\
\hline Modulation techniques & BPSK,QPSK,QAM 16 \\
\hline Channel model & Rician with K=1,5db \\
\hline Diversity combining & MRC, EGC \\
\hline Number of transmitter & $\mathbf{1}$ \\
\hline Number of Receiver & $\mathbf{1 , 2}$ \\
\hline
\end{tabular}

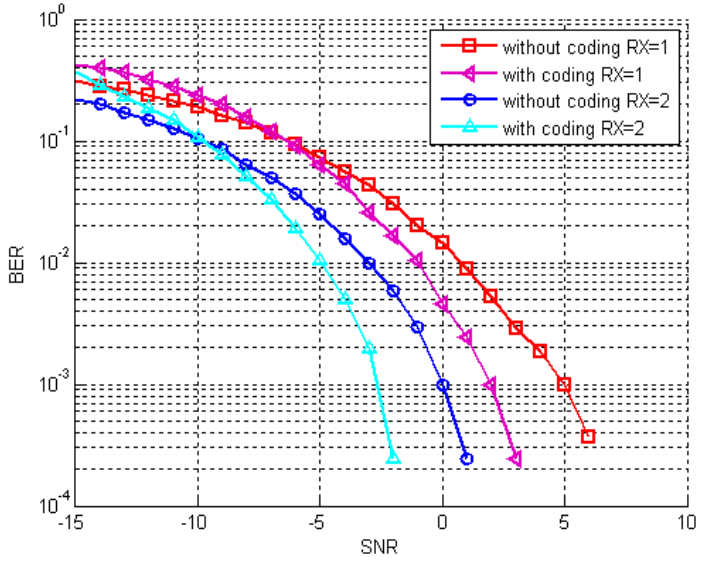

Fig 6: BER performance of MRC using BPSK modulation with rate $1 / 2$ convolutional coding and without convolutional coding for a DS-CDMA system over Rician channel with $K=1 d b$

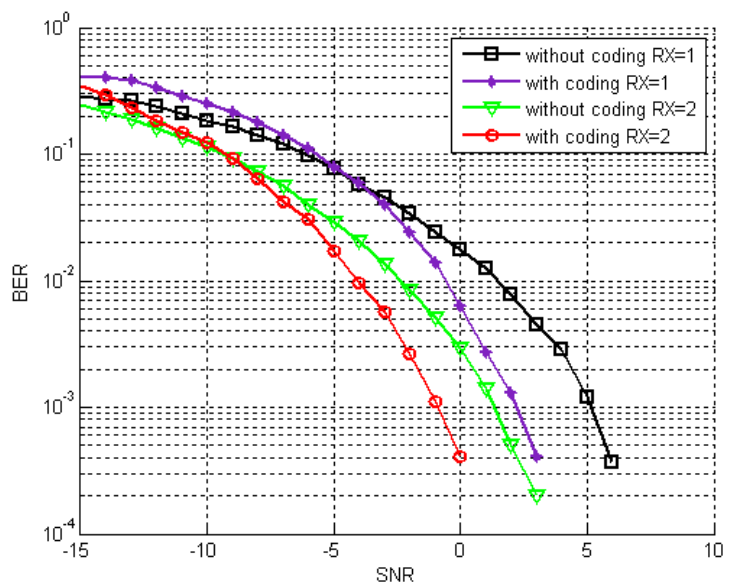

Fig 7: BER performance of EGC using BPSK modulation with rate $1 / 2$ convolutional coding and without convolutional coding for a DS-CDMA system over Rician channel with $K=1 d b$

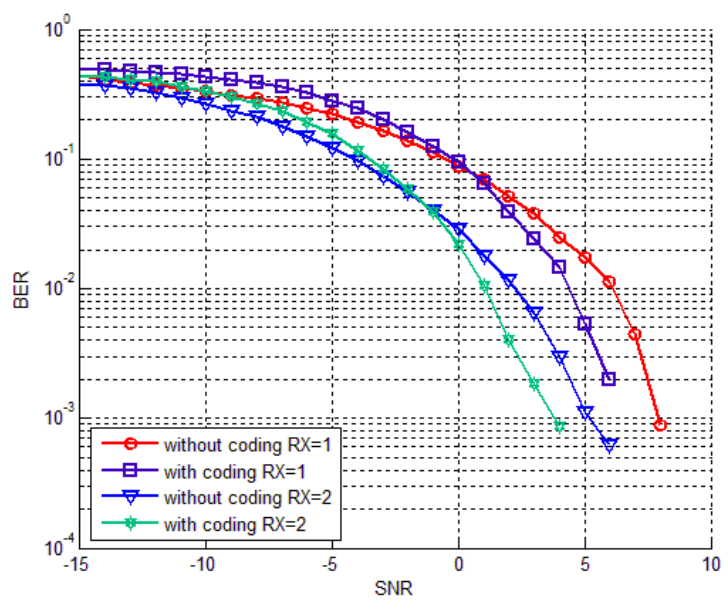

Fig 8: BER performance of MRC using QPSK modulation with rate $1 / 2$ convolutional coding and without convolutional coding for a DS-CDMA system over Rician channel with $K=1 d b$ 


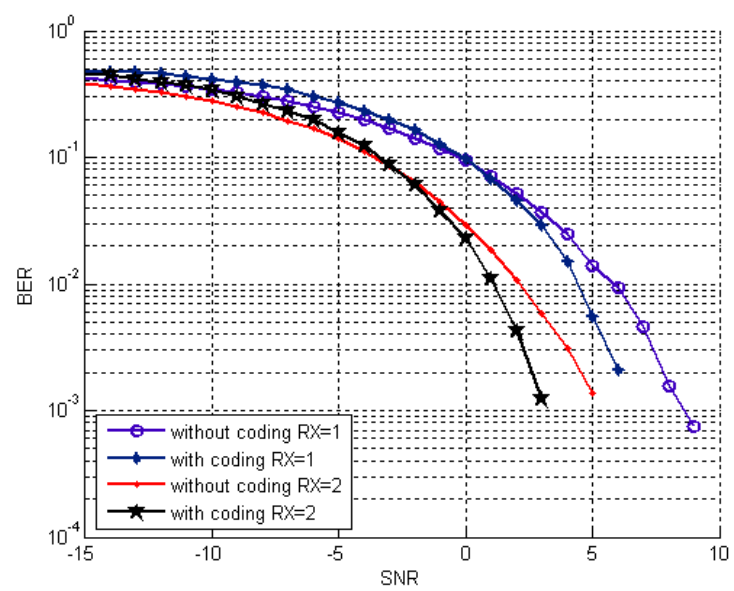

Fig 9: BER performance of EGC using QPSK modulation with rate $1 / 2$ convolutional coding and without convolutional coding for a DS-CDMA system over Rician channel with $K=1 d b$

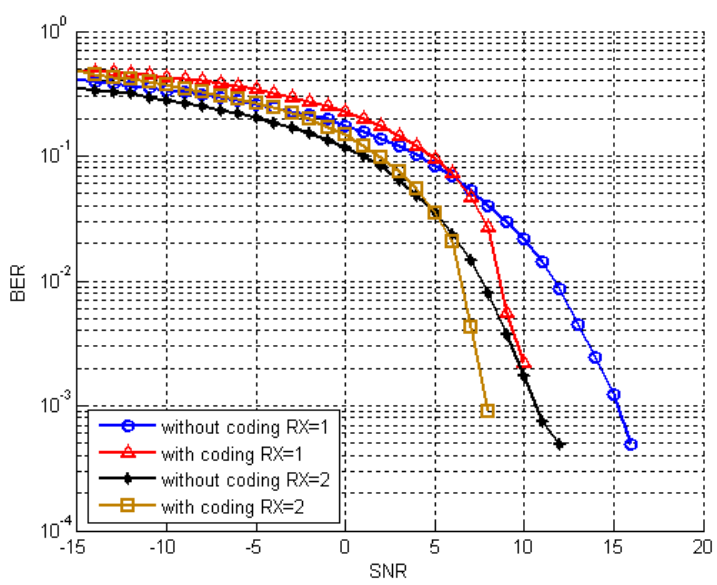

Fig 10: BER performance of MRC using QAM-16 modulation with rate $1 / 2$ convolutional coding and without convolutional coding for a DS-CDMA system over Rician channel with $K=1 d b$

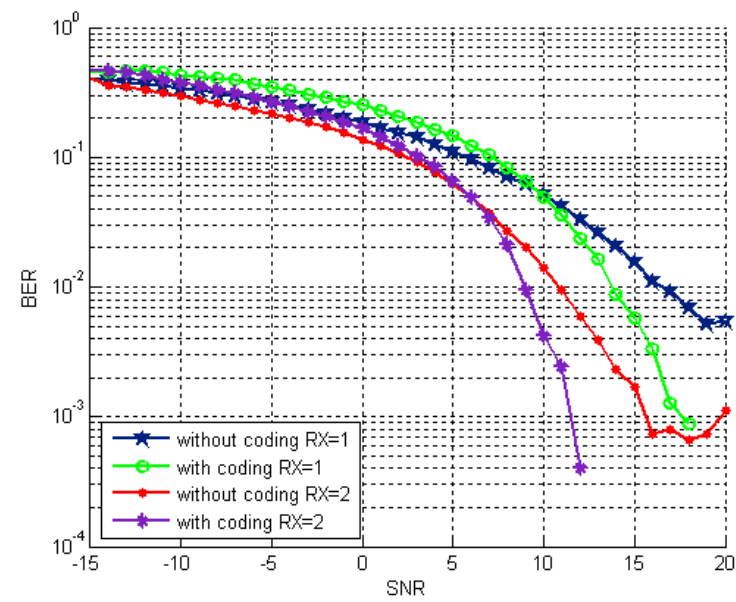

Fig 11: BER performance of EGC using QAM-16 modulation with rate $1 / 2$ convolutional coding and without convolutional coding for a DS-CDMA system over Rician channel with $K=1 d b$

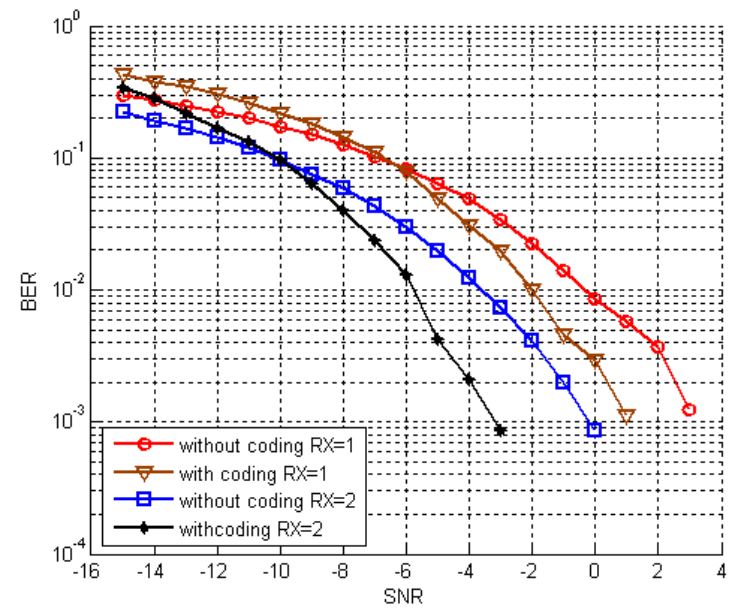

Fig 12: BER performance of MRC using BPSK modulation with rate $1 / 2$ convolutional coding and without convolutional coding for a DS-CDMA system over Rician channel with $K=5 d b$

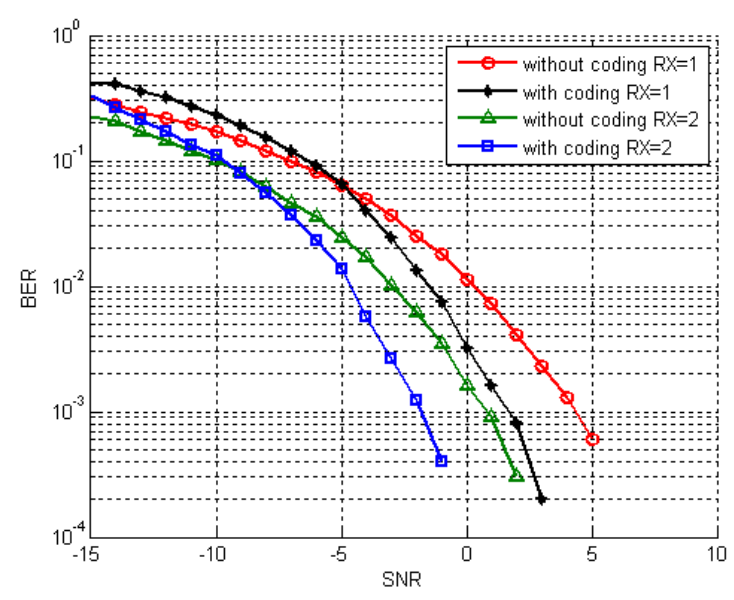

Fig 13: BER performance of EGC using BPSK modulation with rate $1 / 2$ convolutional coding and without convolutional coding for a DS-CDMA system over Rician channel with $K=5 d b$

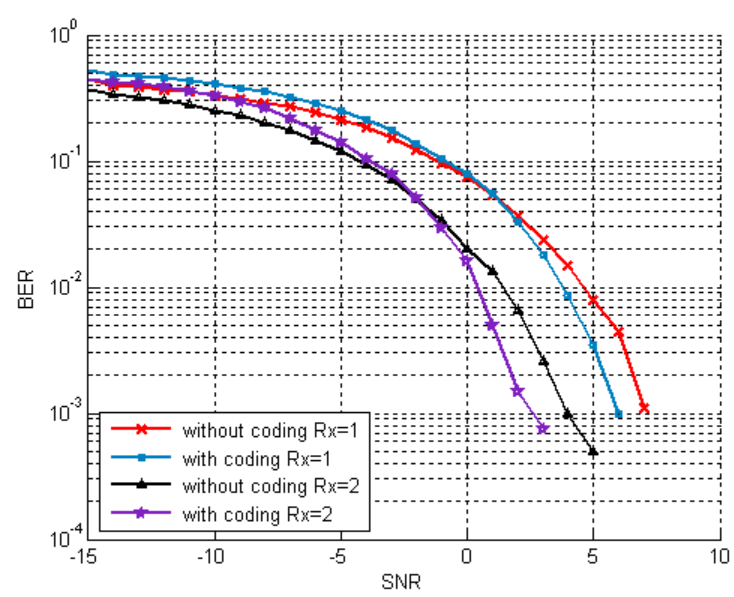

Fig 14: BER performance of MRC using QPSK modulation with rate $1 / 2$ convolutional coding and without convolutional coding for a DS-CDMA system over Rician channel with $K=5 d b$ 


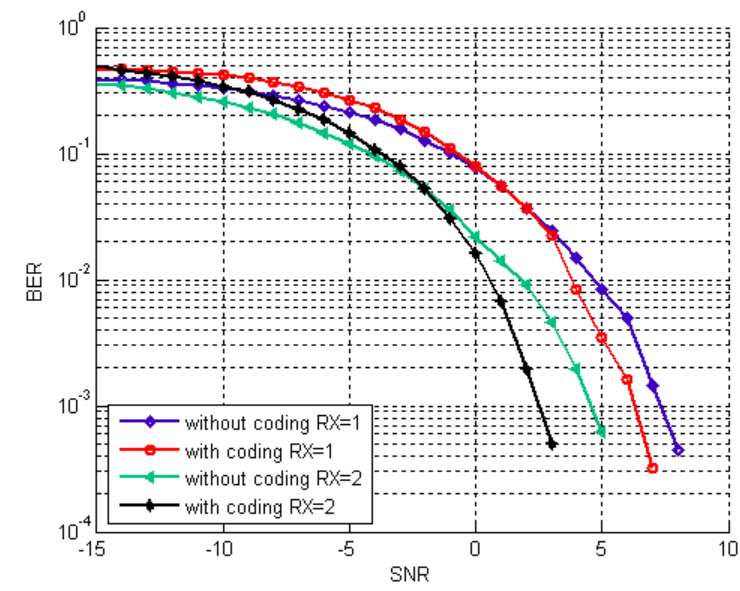

Fig 15: BER performance of EGC using QPSK modulation with rate $1 / 2$ convolutional coding and withou convolutional coding for a DS-CDMA system over Rician channel with $K=5 d b$

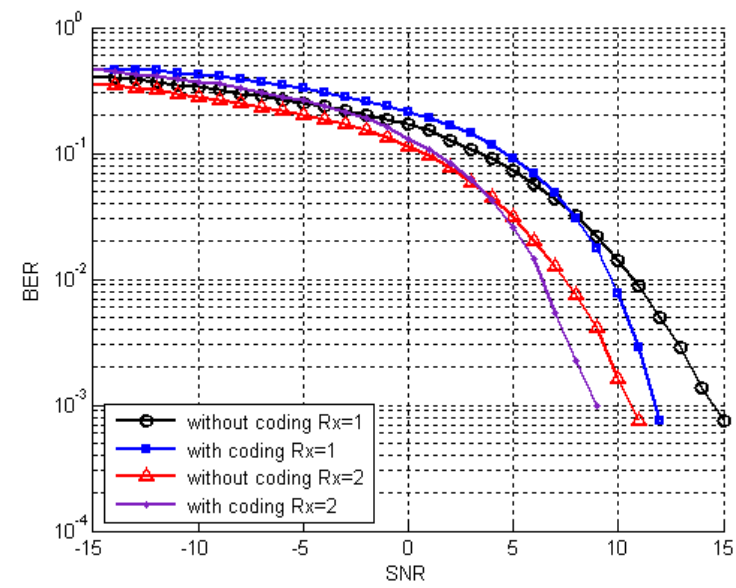

Fig 16: BER performance of MRC using QAM-16 modulation with rate $1 / 2$ convolutional coding and without convolutional coding for a DS-CDMA system over Rician channel with $K=5 d b$

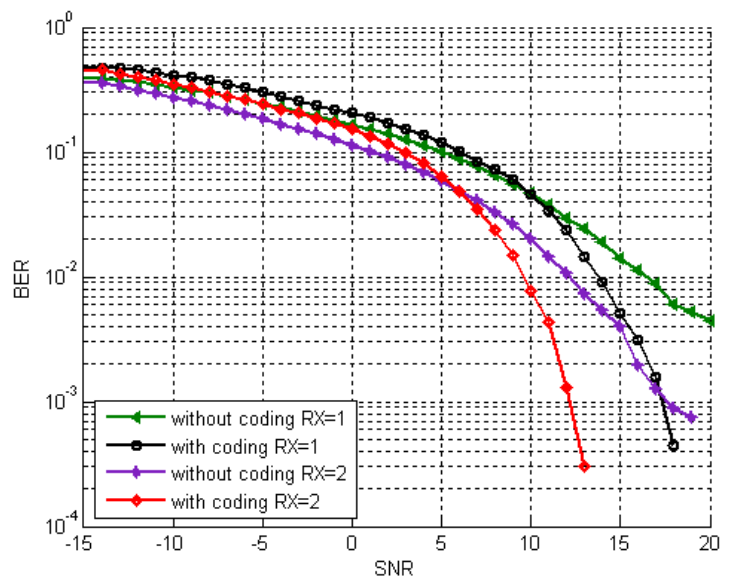

Fig 17: BER performance of EGC using QAM-16 modulation with rate $1 / 2$ convolutional coding and without convolutional coding for a DS-CDMA system over Rician channel with $K=5 d b$

\section{CONCLUSION}

Based on the Simulation results we have concluded that when $1 / 2$ rate convolutional coding is used with different modulation schemes SNR performance of a DS-CDMA system is improved. Among all the three modulation techniques BPSK schemes with $1 / 2$ rate convolutional coding provide more than $5 \mathrm{db}$ gain in SNR performance compare to the QPSK and more than $10 \mathrm{db}$ gain in SNR performance compare to the QAM-16 for a DS-CDMA system with diversity reception. Simulation results also shows that with MRC diversity reception SNR performance of a DS-CDMA system is improved by almost $1 \mathrm{db}$ as compared to the EGC diversity reception. We have also concluded that performance of a DS-CDMA system over Rician channel is also improved by almost $1 \mathrm{db}$ as the value of Rician parameter $\mathrm{K}$ is increases from 1 to 5 .

\section{FUTURE SCOPE}

Work presented in this paper can be extended to evaluate the performance of $1 / 2$ rate convolutional coding for orthogonal frequency division multiplexing (OFDM) system and multicarrier code division multiple access (MC-CDMA) system. Our results can also be extended to include the performance of DS-CDMA system with different modulation schemes using $1 / 2$ rate convolutional coding over Rayleigh, Nakagami and generalized fading channel.

\section{REFERECES}

[1] Mohamed Slim Alouini, Sang Wu Kim and Andrea Goldsmith "RAKE reception with maximal-ratio and equal-gain combining for DS-CDMA systems in Nakagami fading" IEEE 6th International Conference on Universal Personal Communications Record, Vol.2, pp.708 - 712,Oct 1997.

[2] Ramesh Annavajjala, A. Chockalingam and Laurence B. Milstein "Performance Analysis of Coded Communication Systems on Nakagami Fading Channels With Selection Combining Diversity" IEEE Transactions on Communications, Vol. 52, pp.1214-1220, 2004.

[3] Y.L. Chen and C.H. Wei "On the performance of rate $1 / 2$ convolutional codes with QPSK on Rician fading channels" IEEE Transactions on Vehicular Technology,Vol.39, pp.161 - 170,May 1990.

[4] Mischa Schwartz, William R. Bennett and Seymour Stein "Communication Systems and Techniques" IEEE Press, 1996.

[5] A. Annamalai, C. Tellambura and V. K. Bhargava "Unified Analysis of Equal-Gain Diversity on Rician and Nakagami Fading Channels" IEEE WCNC Vol.1, pp.1014, 1999.

[6] Andrea Goldsmith "Wireless Communications" Cambridge University Press, 2005.

[7] Theodore S. Rappaport "Wireless Communication Principal and Practice" Second Edition, Inc. Pearson ed., 2002.

[8] P. Mohana Shankar "Fading and Shadowing in Wireless Systems" Springer, 2012.

[9] M. K. Simon and M.-S. Alouini "Digital Communication over Fading Channels" 1st Ed. New York: Wiley 2000. 